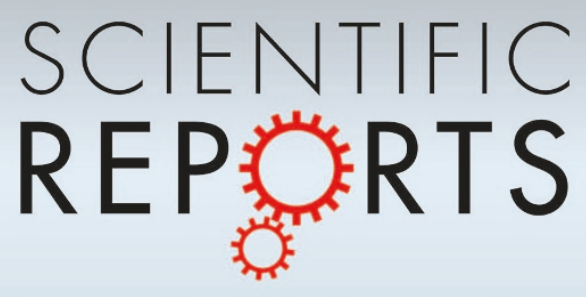

OPEN

SUBJECT AREAS:

SUPERCONDUCTING PROPERTIES AND

MATERIALS

SURFACES, INTERFACES AND THIN FILMS

Received

17 October 2013

Accepted

21 March 2014

Published

10 April 2014

Correspondence and requests for materials should be addressed to V.F.S. (solov@bnl.gov) or Q.L. (qiangli@bnl.

gov)

\title{
Highly efficient solid state catalysis by reconstructed (001) Ceria surface
}

\author{
Vyacheslav F. Solovyov' ${ }^{1}$, Toshinori Ozaki ', Andrea Atrei ${ }^{2}$, Lijun Wu' ${ }^{1}$, Abdullah Al-Mahboob ${ }^{3}$, \\ Jerzy T. Sadowski ${ }^{3}$, Xiao Tong ${ }^{3}$, Dmytro Nykypanchuk ${ }^{3} \&$ Qiang Li ${ }^{1}$
}

\begin{abstract}
${ }^{1}$ Condensed Matter Physics and Materials Science Department, Brookhaven National Laboratory, Upton, NY 11973 , ${ }^{2}$ Dipartimento di Biotecnologie, Chimica e Farmacia, Università di Siena, 53100 Siena, Italy, ${ }^{3}$ Center for Functional Nanomaterials, Brookhaven National Laboratory, Upton, NY 11973.
\end{abstract}

Substrate engineering is a key factor in the synthesis of new complex materials. The substrate surface has to be conditioned in order to minimize the energy threshold for the formation of the desired phase or to enhance the catalytic activity of the substrate. The mechanism of the substrate activity, especially of technologically relevant oxide surfaces, is poorly understood. Here we design and synthesize several distinct and stable $\mathrm{CeO}_{2}(001)$ surface reconstructions which are used to grow epitaxial films of the high-temperature superconductor $\mathrm{YBa}_{2} \mathrm{Cu}_{3} \mathrm{O}_{7}$. The film grown on the substrate having the longest, fourfold period, reconstruction exhibits a twofold increase in performance over surfaces with shorter period reconstructions. This is explained by the crossover between the nucleation site dimensions and the period of the surface reconstruction. This result opens a new avenue for catalysis mediated solid state synthesis.

E pitaxial coatings and interfaces of complex superconducting materials often exhibit properties far superior to those of their bulk counterparts ${ }^{1-3}$. Novel material properties are expected to arise from structures grown on engineered substrates. The substrate surface catalytic activity is defined as the ability of the surface to reduce the nucleation energy barrier, thus promoting the formation of desirable phases and suppressing secondary reactions $s^{4,5}$. The activity of a substrate is becoming increasingly important. As the materials become more complex, competition from secondary phases becomes an acute problem. The traditional approach has utilized an empirical search for compatible substrate materials ${ }^{6}$. Purposeful engineering of the substrate surface may allow for high performance films using robust and practical simple oxide templates.

$\mathrm{CeO}_{2}$, ceria is a promising simple oxide ${ }^{7}$. Its high melting temperature and cubic $f c c$ (fluorite) structure make it an ideal substrate. A bulk (001) termination of ceria is charged (polar), which means that it undergoes a stabilization process by reconstruction and/or relaxation ${ }^{8}$. Stable non-polar surfaces of cubic oxides, such as (001) for the rock salt-type $(\mathrm{MgO}, \mathrm{NiO})$ and $(111)$ for fluorite-type $\left(\mathrm{CeO}_{2}\right)$ received much attention over the last several decades, being the subjects of intense experimental studies by a variety of techniques? ${ }^{9}$. Polar surfaces of epitaxial films, such as (111) of $\mathrm{MgO}$ and (001) of $\mathrm{CeO}_{2}$ remain poorly explored.

In this work we manipulate the stabilization process in an effort to enhance the catalytic activity of the (001) ceria surface. We employ low-energy electron diffraction (LEED) and X-ray surface diffraction in order to relate the structure of the (001) ceria surface to its ability to nucleate high-temperature superconducting $\mathrm{YBa}_{2} \mathrm{Cu}_{3} \mathrm{O}_{7}$ (YBCO) during an ex-situ growth process ${ }^{10}$. The ex-situ growth process involves a chemical reaction, a reduction of the fluorinated precursor ${ }^{11}$. The precursor reduction is a rate-limiting step with requires a catalytically active substrate at the YBCO nucleation stage. We show that the nucleation process is strongly affected by the symmetry and periodicity of the surface structure of the (001) ceria termination.

\section{Results}

The $70 \mathrm{~nm}$ thick (001) ceria films were deposited on (001) yttrium-stabilized zirconia (YSZ) substrates by pulsed laser deposition, followed by annealing for $30 \mathrm{~min}$ at $900^{\circ} \mathrm{C}$ in various environments as follows: $\left.i\right) \mathrm{Sample} \mathrm{Ce}\left(\mathrm{H}_{2}-\right.$ $\mathrm{H}_{2} \mathrm{O}$ ): Mixture of $3.5 \% \mathrm{H}_{2}$, balance Argon saturated with water vapor; the mixture included $\mathrm{H}_{2}$ and $\mathrm{H}_{2} \mathrm{O}$ partial pressures at approximately equal values, $p \mathrm{H}_{2} \approx p \mathrm{H}_{2} \mathrm{O} \approx 25$ Torr; ii) Sample $\mathrm{Ce}\left(\mathrm{H}_{2} \mathrm{O}\right): 20$ Torr of water vapor; iii) Sample $\mathrm{Ce}\left(\mathrm{O}_{2}\right): 100 \%$ Oxygen. The samples were cooled down in the processing atmosphere and exposed to the ambient during the transfer for the LEED measurement.

Fig. 1 compares LEED reciprocal space maps (RSM) and AFM height maps of an as-grown sample (panels (a) and (c)) to that of a sample processed for $30 \mathrm{~min}$ at $900^{\circ} \mathrm{C}$ in $3.5 \% \mathrm{H}_{2}$ - $\mathrm{Ar}$ mix saturated with water vapor (panels 


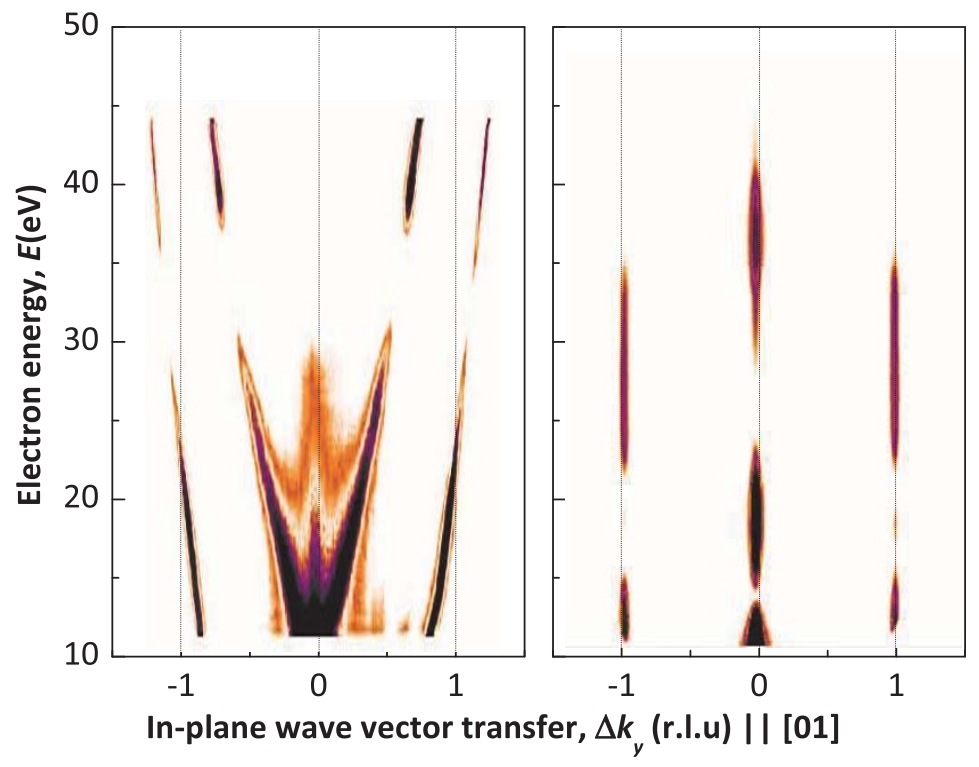

(c) As-deposited

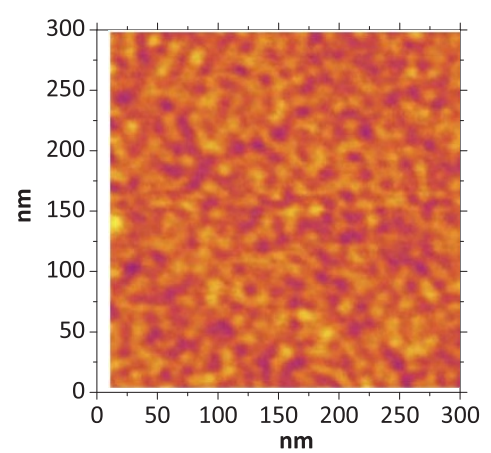

(d) Annealed in $\mathrm{H}_{2}-\mathrm{H}_{2} \mathrm{O}$

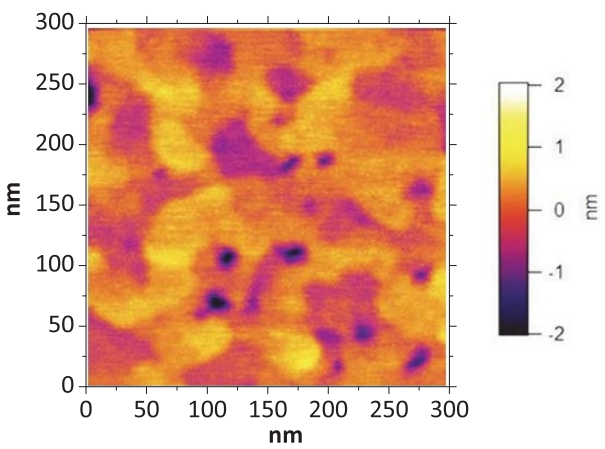

Figure 1 Surface morphology transformation of the as-deposited $\mathrm{CeO}_{2}$ buffer after post-annealing. Reciprocal space LEED maps (a,b) and AFM height maps $(\mathrm{c}, \mathrm{d})$ demonstrate the transition from a small-grain surface of the as-deposited sample $(\mathrm{a}, \mathrm{c})$ to flat-top, large grain structure after the annealing in $\mathrm{H}_{2}-\mathrm{H}_{2} \mathrm{O}$ mixture for $30 \mathrm{~min}$ at $900^{\circ} \mathrm{C}$, (b,d).

(b) and (d)). The Atomic Force Microscopy (AFM) height demonstrates a typical transformation of a rough as-deposited surface of $\mathrm{CeO}_{2}$ into a conglomeration of flat-top grains. The corresponding LEED RSMs are shown in Fig. 1a,b. The RSM of the as-deposited sample is comprised of diverging streaks (Fig. 1a) which become vertical lattice rods after annealing (Fig. 1b) indicating formation of a structurally-ordered flat surface. The X-ray reflectivity detected a change of surface roughness from $2.5 \mathrm{~nm}$ in the as-deposited sample to $0.5-0.3 \mathrm{~nm}$ after annealing, thus confirming that postannealing produces dense films with less than unit cell roughness (Supplementary Fig. 1Sa).

We determined the principal surface lattice parameter of all the three samples using LEED patterns, the Ag (001) surface was utilized as the calibration standard. These lattice parameters are $0.37 \pm$ $0.02 \mathrm{~nm}$, which is within the experimental error of the lattice constant of a bulk surface, $0.38 \mathrm{~nm}$. The in-plane and out-of-plane bulk lattice parameters, measured by X-ray diffraction, were observed to be $0.542 \pm 0.03 \mathrm{~nm}$, with the sample-to-sample variation within the experimental error. The bulk structure of the film was shown to be consistent with cubic cerium oxide $\mathrm{CeO}_{\mathrm{x}}$ with $x \approx 2$. The $\mathrm{X}$-ray photoemission spectroscopy (XPS) analysis of samples $\mathrm{Ce}\left(\mathrm{H}_{2}\right.$ $\left.\mathrm{H}_{2} \mathrm{O}\right), \mathrm{Ce}\left(\mathrm{O}_{2}\right)$, and $\mathrm{Ce}\left(\mathrm{H}_{2} \mathrm{O}\right)$ detected $\approx 10 \% \mathrm{Ce}^{3+}$, independently confirming predominantly $\mathrm{Ce}^{4+}$ surface oxidation state (see
Supplementary Fig. 1Sb,c,d). The XPS experiment was repeated after the LEED measurement, which also included annealing the sample at $600^{\circ} \mathrm{C}$ for $30 \mathrm{~min}$ in a UHV environment prior to the XPS spectra acquisition. The XPS spectra remained unchanged, confirming that the UHV exposure at $600^{\circ} \mathrm{C}$ did not affect the oxidation state.

Fig. 2 is a side-by-side presentation of the LEED patterns (averaged over fixed energy patterns acquired in the 10-45 eV range) for the samples a) $\mathrm{Ce}\left(\mathrm{H}_{2}-\mathrm{H}_{2} \mathrm{O}\right)$, b) $\mathrm{Ce}\left(\mathrm{H}_{2} \mathrm{O}\right)$ and c) $\mathrm{Ce}\left(\mathrm{O}_{2}\right)$. In the following discussion we use the $2 \mathrm{D}$ surface cell of the unreconstructed (001) Ceria surface (lattice constant $0.38 \mathrm{~nm}$ ) as the reference structure, the reconstructions being defined as modifications of the surface cell. Boundaries of the first Brillouin zone (BZ) of the unreconstructed surface cell are indicated by dashed lines. The sections of the first (BZ) along [01] and [11] directions are shown in panels Fig. 2 d,e and Fig. 2f,g, respectively. The diffraction pattern of the $\mathrm{Ce}\left(\mathrm{H}_{2}-\mathrm{H}_{2} \mathrm{O}\right)$ sample indicates an unreconstructed surface. The RSMs of this sample (Fig. 2d,f) reveal only the first order (01) and (11) reflections. In contrast, the LEED patterns from samples $\mathrm{Ce}\left(\mathrm{H}_{2} \mathrm{O}\right)$ and $\mathrm{Ce}\left(\mathrm{O}_{2}\right)$ contain well-defined fractional order reflections, some indicated by arrows in Fig. 2b,c. The corresponding RSMs in Fig. 2e,g demonstrate that positions of the fractional reflections do not depend on the electron beam energy. This rules out a contribution by surface faceting. 
(a) $\mathrm{Ce}\left(\mathrm{H}_{2}-\mathrm{H}_{2} \mathrm{O}\right)$

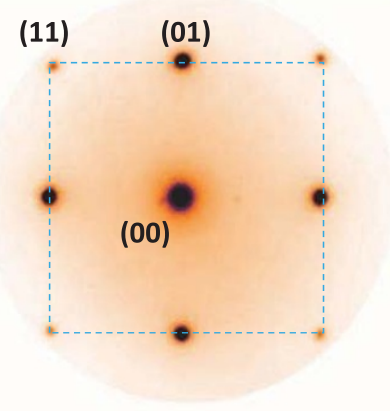

(b) $\mathrm{Ce}\left(\mathrm{H}_{2} \mathrm{O}\right)$

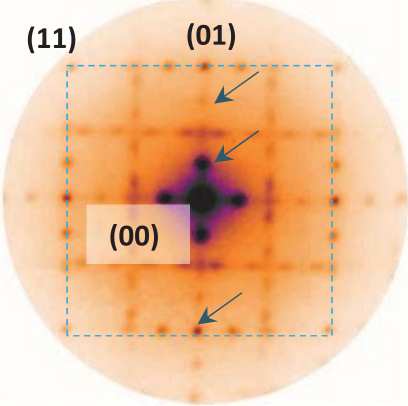

(c) $\mathrm{Ce}\left(\mathrm{O}_{2}\right)$ (d) $\mathrm{Ce}\left(\mathrm{H}_{2}-\mathrm{H}_{2} \mathrm{O}\right)$ (f) $\mathrm{Ce}\left(\mathrm{H}_{2}-\mathrm{H}_{2} \mathrm{O}\right)$

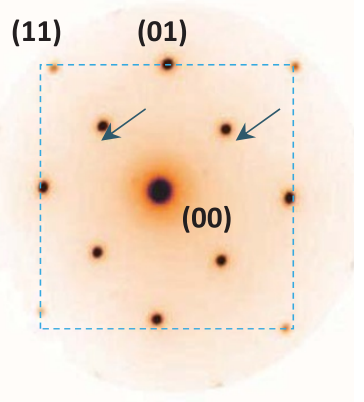

(g) $\mathrm{Ce}\left(\mathrm{O}_{2}\right)$
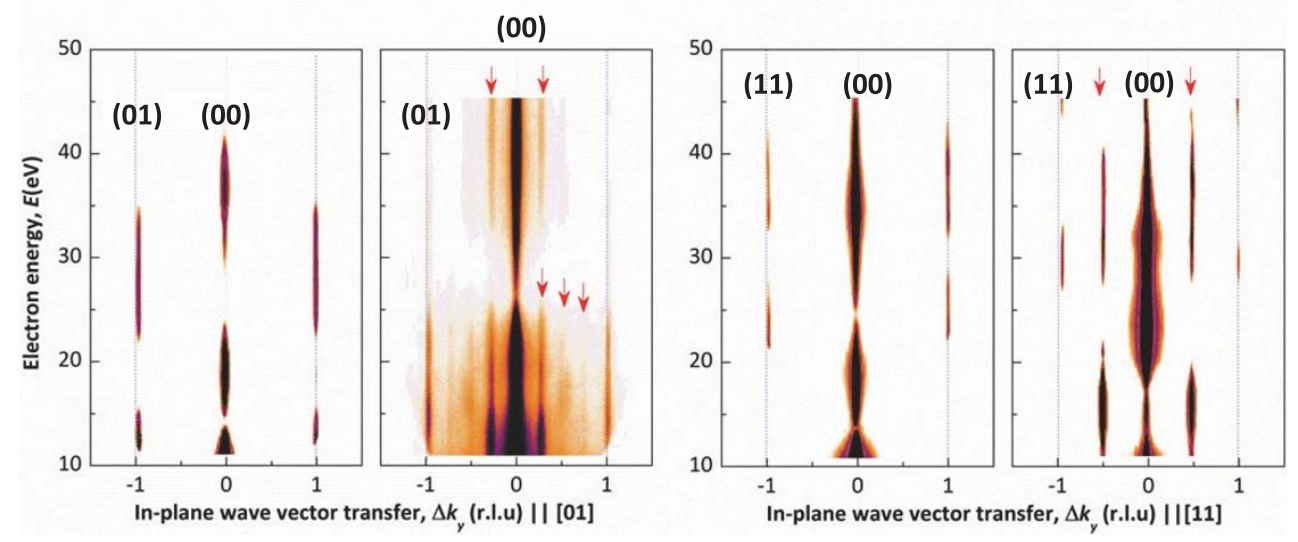

Figure $2 \mid$ Reconstructions of (001) ceria surface. LEED diffraction patterns of samples (a) $\mathrm{Ce}\left(\mathrm{H}_{2}-\mathrm{H}_{2} \mathrm{O}\right) ;(\mathrm{b}) \mathrm{Ce}\left(\mathrm{H}_{2} \mathrm{O}\right)$ and $(\mathrm{c}) \mathrm{Ce}\left(\mathrm{O}_{2}\right)$. The patterns show distinct reconstruction reflections, some pointed out by arrows. (d-f) Panels (d) and (f) compare the reciprocal space sections of the apparently unreconstructed surface of the sample $\mathrm{Ce}\left(\mathrm{H}_{2}-\mathrm{H}_{2} \mathrm{O}\right)$ with that of samples $\mathrm{Ce}\left(\mathrm{H}_{2} \mathrm{O}\right)$ (panel e) and $\mathrm{Ce}\left(\mathrm{O}_{2}\right)$ (panel g). The arrows indicate fractional reflections. The dashed lines in the LEED patterns outline the first BZ.

With the exception of the $\mathrm{Ce}\left(\mathrm{H}_{2}-\mathrm{H}_{2} \mathrm{O}\right)$ sample, the LEED patterns in Fig. 2 did not change as the sample was cooled from $600^{\circ} \mathrm{C}$. Fig. 3 shows the change of the LEED pattern of the $\mathrm{Ce}\left(\mathrm{H}_{2}-\mathrm{H}_{2} \mathrm{O}\right)$ sample upon cooling from (a) $600^{\circ} \mathrm{C}$ to (b) $300^{\circ} \mathrm{C}$. The pattern at $600^{\circ} \mathrm{C}$ contains the primary reflections of the first BZ. The emergence of multiple fractional reflections was observed during the cooling, $1 / 2$ order being the most prominent. Additionally, we observe several fractional reflections around the principal (01) peak.

We conducted several experiments aimed at assessing the stability of the surface reconstructions to oxidation. Supplementary Fig. $2 \mathrm{~S}$ compares LEED patterns of sample $\mathrm{Ce}\left(\mathrm{H}_{2} \mathrm{O}\right)$ before and after the oxidation at $870^{\circ} \mathrm{C}$ in $300 \mathrm{mTorr}$ of oxygen for $30 \mathrm{~min}$. The pattern retained the characteristic fractional reflections, confirming the oxidation resistance of the surface structure. Complementary surface characterization using differentially pumped Reflection HighEnergy Electron Diffraction (RHEED) documented the effect of oxygen exposure on the surface structure. Supplementary Fig. 3S compares LEED and RHEED patterns of $\mathrm{Ce}\left(\mathrm{H}_{2} \mathrm{O}\right)$ sample. The RHEED pattern was acquired at $5 \times 10^{-6}$ Torr of oxygen partial pressure, $600^{\circ} \mathrm{C}$, which is well within $\mathrm{Ce}^{4+}$ stability range ${ }^{9}$. The presence of well-defined fractional reflection in the RHEED pattern suggests that the surface structure is comprised of $\mathrm{Ce}^{4+}$ ions.

Fig. 4a-c compares the nucleation density of $0.8 \mu \mathrm{m}$ thick YBCO superconductor films deposited on the three reconstructed substrates used in the study. The YBCO grains can be seen as dark circles on a light background; a secondary $\mathrm{Ba}-\mathrm{Cu}-\mathrm{O}$ phase conglomerate is shown as grayish areas. The phases were identified by cross-referencing the optical images with the Raman spectra. We observe that the $\mathrm{Ce}\left(\mathrm{H}_{2} \mathrm{O}\right)$ sample has the highest activity, which corresponds to the highest density of YBCO grains. Fig. 4d,e shows a comparison of the field dependences of the critical current density at 77 and $30 \mathrm{~K}$ (a) $\mathrm{Ce}\left(\mathrm{H}_{2}-\mathrm{H}_{2} \mathrm{O}\right)$ at $600^{\circ} \mathrm{C}$

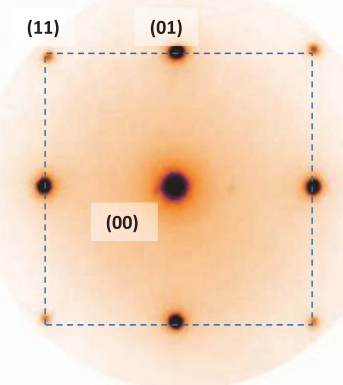

(b) $\mathrm{Ce}\left(\mathrm{H}_{2}-\mathrm{H}_{2} \mathrm{O}\right)$ at $300^{\circ} \mathrm{C}$

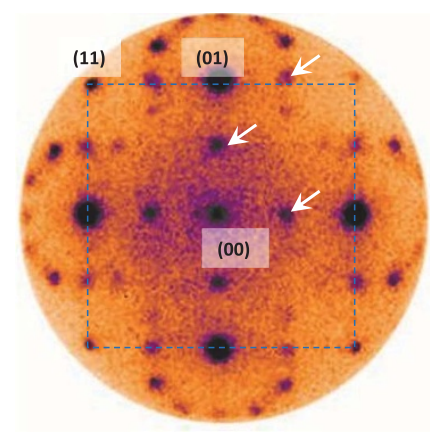

Figure $3 \mid$ Ordering of $\mathrm{Ce}\left(\mathrm{H}_{2}-\mathrm{H}_{2} \mathrm{O}\right)$ sample at $300^{\circ} \mathrm{C}$. LEED diffraction patterns of $\mathrm{Ce}\left(\mathrm{H}_{2}-\mathrm{H}_{2} \mathrm{O}\right)$ sample (a) at $600^{\circ} \mathrm{C}$ and $(\mathrm{b})$ at $300^{\circ} \mathrm{C}$. The fractional reflections are labeled with arrows. The dashed lines outline the first BZ. 
(a) $\mathrm{Ce}\left(\mathrm{H}_{2}-\mathrm{H}_{2} \mathrm{O}\right)$

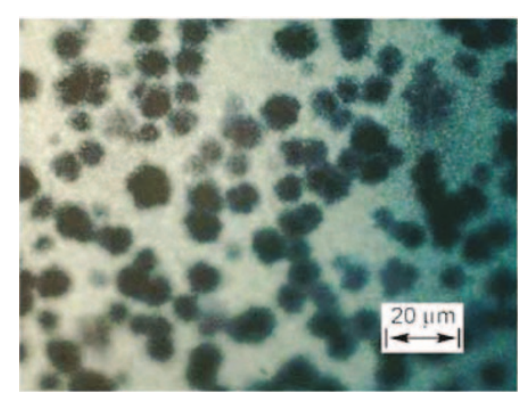

(b) $\mathrm{Ce}\left(\mathrm{H}_{2} \mathrm{O}\right)$



(c) $\mathrm{Ce}\left(\mathrm{O}_{2}\right)$

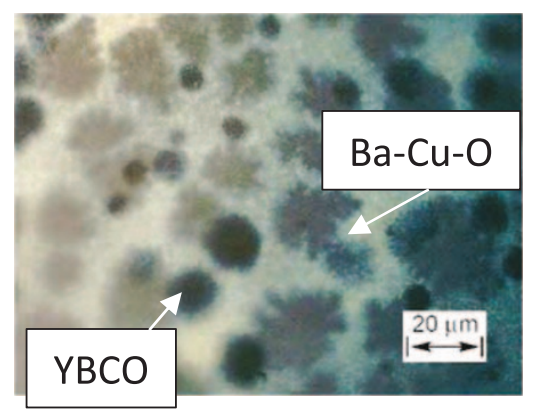

(d) $77 \mathrm{~K}$



(e) $30 \mathrm{~K}$



Figure $4 \mid$ Effect of the surface reconstruction on the substrate activity. Optical micrograph of YBCO grains (dark disks) and Ba-Cu-O secondary phase conglomerates (grayish areas) embedded in a precursor film deposited on (a) $\mathrm{Ce}\left(\mathrm{H}_{2}-\mathrm{H}_{2} \mathrm{O}\right),\left(\right.$ b) $\mathrm{Ce}\left(\mathrm{H}_{2} \mathrm{O}\right)$ and $(\mathrm{c}) \mathrm{Ce}\left(\mathrm{O}_{2}\right)$ buffers. Panels $(\mathrm{d})$ and $(e)$ compare field dependences of the critical current density at $77 \mathrm{~K}$ and $30 \mathrm{~K}$ correspondingly.

correspondingly. The film deposited on $\mathrm{Ce}\left(\mathrm{H}_{2} \mathrm{O}\right)$ substrates demonstrates approximately twice the critical current density of $\mathrm{Ce}\left(\mathrm{H}_{2}\right.$ $\left.\mathrm{H}_{2} \mathrm{O}\right)$ or $\mathrm{Ce}\left(\mathrm{O}_{2}\right)$ substrates, both at 77 and $30 \mathrm{~K}$.

\section{Discussion}

Cerium oxide is a compound with high melting temperature $\left(\sim 2500^{\circ} \mathrm{C}\right)$. This explains the very limited mobility of Ce atoms at practical deposition temperatures $\left(<1000^{\circ} \mathrm{C}\right)$. Typical as-deposited Ceria films can be described as Zone 2 films using Thornton's classification scheme ${ }^{12}$. Zone 2 films are comprised of dense arrays of columnar grains with faceted surfaces and voided grain boundaries. Since the (111) surface has the lowest energy in a wide range of oxidation potentials $\left(1.41 \mathrm{~J} / \mathrm{m}^{2} \text { for (111) vs. } 0.68 \mathrm{~J} / \mathrm{m}^{2} \text { for }(100)\right)^{13}$, columnar (001) $\mathrm{CeO}_{2}$ films are expected to be terminated by pyramid-like (111) terminations. Such surface structures were indeed observed by electron and tunneling microscopy methods ${ }^{14,15}$. A faceted surface is identified in a LEED experiment via the dependence of the diffraction peak positions in the lateral reciprocal space coordinates, $k_{x-y}$, on the incident beam energy $E^{16}$.

Fig. 1 shows the juxtaposition of an AFM height map of an asdeposited sample with its $k_{x-y}-E$ RSM. One can clearly see that cusp-like terminations of the Ceria grains in as-deposited film produce energy-dependent reflections which appear as tilted rods on the map. Post-annealing causes lateral grain growth with the formation of flat (001) terminations which replace cusp-like (111) facets ${ }^{17}$. This change in morphology is reflected in significant smoothing of the surface roughness and development of vertical lattice rods on the reciprocal space map (Fig. 1b). Ceria films on YSZ exhibit classical normal grain growth ${ }^{18}$. The lateral grain growth is checked by the thermal grooving effect ${ }^{19}$ when the grain size becomes approximately equal to the film thickness. The final film morphology is the result of a balance between grain boundary energy and energy of the free surface. As a result, (001) terminations, which are practically absent in a free standing Ceria nano-crystal ${ }^{20}$, become dominant features of the film surface (Fig. 1d) ${ }^{21,22}$.

Fig. 2 exhibits the central result of the paper. The origin of the surface reconstruction variety comes from the polar nature of the (001) surface of ceria ${ }^{23}$. An unreconstructed polar surface is intractable due to the infinite energy associated with the non-zero electric field $^{23}$. The infinite energy problem can be resolved by removing $50 \%$ of the anions from one of the terminations (effectively placing them on the other side of the slab $)^{24}$, which eliminates the internal electric field and renders the surface non-polar ${ }^{25}$. Indeed, a study of the (001) Ceria surface by the angle-resolved mass spectroscopy of recoiled ions has shown that half of the oxygen ions are missing from the (001) termination ${ }^{26}$. Partial reduction of the surface Cerium to $3+$ would require more than $50 \%$ oxygen desorption in order to maintain the charge neutrality. The oxidation levels used to heat treat the sample are well within the $\mathrm{Ce}^{4+}$ stability range $\mathrm{e}^{27}$, which is corroborated by XPS, X-ray diffraction and recent density functional theory (DFT) calculations ${ }^{9,28}$. We estimate that up to $25 \%$ of $\mathrm{Ce}^{3+}$ ions in the top layer would not be detected by XPS, which sets the lower range of the surface oxygen coverage, $\theta$, at 0.45 .

Once $\approx 50 \%$ of the oxygen atoms are desorbed, ordering of the remaining atoms can be described by lattice gas theory ${ }^{29,30}$ as an absorbate on a square lattice with the average absorption site occupancy $\theta \approx 0.5$. Lattice gas theory has been successful in predicting the ordering of $\mathrm{H}, \mathrm{O}, \mathrm{S}$ on low-index metal surfaces ${ }^{31}$. An important difference is that the coverage of a metal surface by a gas depends on the chemical potential of the absorption reaction. Coverage of a 
(a) $\mathrm{Ce}\left(\mathrm{H}_{2}-\mathrm{H}_{2} \mathrm{O}\right), p(1 \times 1)$

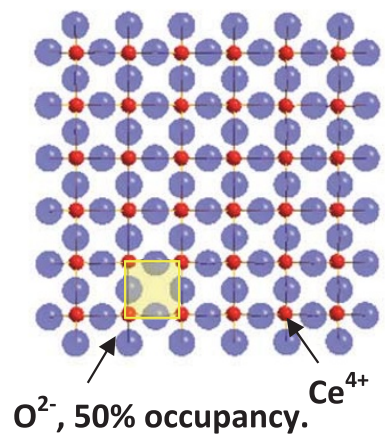

(d) $\mathrm{Ce}\left(\mathrm{H}_{2}-\mathrm{H}_{2} \mathrm{O}\right)$



(g) $\mathrm{Ce}\left(\mathrm{H}_{2}-\mathrm{H}_{2} \mathrm{O}\right), p(1 \times 1)$



(b) $\mathrm{Ce}\left(\mathrm{H}_{2} \mathrm{O}\right), p(4 \times 1)$



(e) $\mathrm{Ce}\left(\mathrm{H}_{2} \mathrm{O}\right)$



(c) $\mathrm{Ce}\left(\mathrm{O}_{2}\right), c(2 \times 1)$

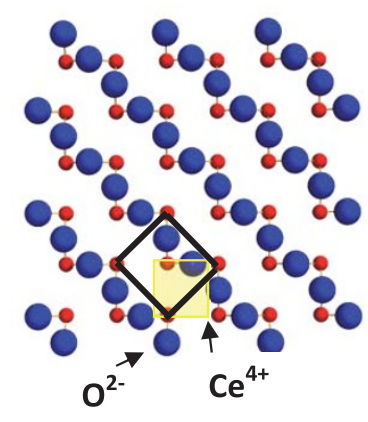

(f) $\mathrm{Ce}\left(\mathrm{O}_{2}\right)$

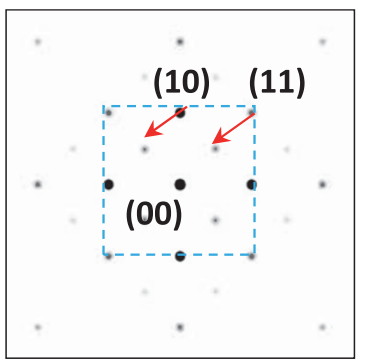

(h) $\mathrm{Ce}\left(\mathrm{O}_{2}\right)$

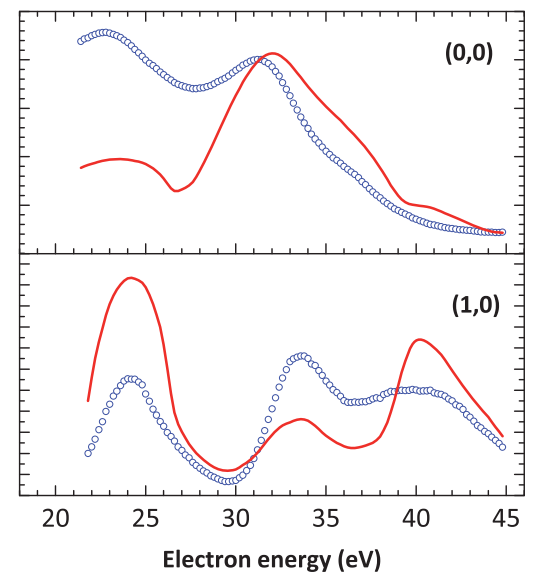

Figure $5 \mid$ Possible surface $\mathrm{O}^{2-}$ ion arrangements of reconstructed $\mathrm{CeO}_{2}(001)$ terminations. The $p(1 \times 1)$ structure, (a), is modeled as random distribution of oxygen vacancies. The bottom row, (d)-(f) presents corresponding diffraction patterns calculated within the kinematic approximation; patterns (e) and (f) are calculated taking into account two orthogonal orientations of domains shown in (b) and (c). The dashed square outlines the first $\mathrm{BZ}$ of the unreconstructed surface. The arrows point out the extra reflections due to oxygen ordering. The shaded square in panels (a)-(c) represents 0.38 $\times 0.38 \mathrm{~nm}$ attachment site of a c-axis oriented YBCO nucleus. In panels (b) and (c) the unit cells are outline by a bold line. Note that $p(4 \times 1)$ domain of the $\mathrm{Ce}\left(\mathrm{H}_{2} \mathrm{O}\right)$ sample offers a structurally perfect attachment site, while $c(2 \times 1)$ and $p(1 \times 1)$ have, on average, two vacancies per site. Panels $\left.\left.\mathrm{g}\right)-\mathrm{h}\right)$ present results of quantitative LEED beam intensity analysis (solid line) of $(00)$ and $(01)$ beams of $p(1 \times 1)$ and $c(2 \times 1)$ arrangements.

reconstructed oxide surface is fixed by the requirement of charge neutrality. In our model we assume that the surface stays oxygen terminated, which is supported by DFT calculations ${ }^{13,32}$. The total interaction energy of the lattice gas is:

$$
H=-\sum_{i \neq j} \varphi_{i j} c_{j} c_{i}-\sum_{i \neq j \neq k} \varphi_{t} c_{j} c_{j} c_{k}
$$

Where $c_{i}$ is the local occupation variable, $c_{i}=1$ if the site $i$ is occupied and zero otherwise, $\varphi_{i j}$ is equal to either $\varphi_{n n}$ for the next neighbor pair or $\varphi_{n n n}$ for a next nearest pair and $\varphi_{t}$ is a three atom interaction term. One can easily show that Eq. 1 is equivalent to a spin-spin interaction term of an Ising magnet ${ }^{33}$.

The simplest case is a purely repulsive interaction between the nearest neighbors: $\varphi_{n n}<0, \varphi_{t}=\varphi_{n n n}=0$. The model predicts ordering of the gas into a $\times 2$ lattice (similar to the Ising antiferromagnetic state) below a critical temperature, $T_{c}$, where $T_{c} \sim \varphi_{n n}$. The ordered state exists in the range of coverage $0.35<\theta<0.64$, with the maximum $T_{c}$ at $\theta=0.5$. Fig. $5 \mathrm{c}$ shows the structure model of the $\times 2$ (001) ceria surface that describes the reconstruction of the $\mathrm{Ce}\left(\mathrm{O}_{2}\right)$ sample. The model diffraction pattern, which assumes equal distribution of $90^{\circ} c(2 \times 1)$ rotational domains, contains characteristic fractional $\left(\begin{array}{ll}1 / 2 & 1 / 2\end{array}\right)$ reflections, consistent with the experimentallyobserved LEED pattern of the $\mathrm{Ce}\left(\mathrm{O}_{2}\right)$ sample, as shown in Fig. 2c. The oxygen vacancy rows in Fig. $5 c$ are parallel to $<100>$ direction which differs from the checkerboard pattern of the low-energy reconstructed (001) surface proposed by Conesa ${ }^{25}$. Even though electrostatic calculations ${ }^{25}$ showed that the the checkerboard pattern has relatively low energy, the diffraction produced by this surface does 
not contain the additional $\left(\begin{array}{ll}1 / 2 & 1 / 2\end{array}\right)$ reflections, observed in the experiment (see Fig. 2c). The $I$ - $V$ curves calculated for this structural model (solid lines) are compared with the experimental ones (open symbols) in Fig. 5h. The results of the LEED intensity analysis suggest that the proposed model is compatible with the experimental data, thus identifying the surface of the $\mathrm{Ce}\left(\mathrm{O}_{2}\right)$ sample as an ordered lattice gas phase with purely repulsive nearest neighbor interaction.

The absence of super-lattice reflections in the LEED pattern from the $\mathrm{Ce}\left(\mathrm{H}_{2}-\mathrm{H}_{2} \mathrm{O}\right)$ sample, Fig. $2 \mathrm{a}$, is then explained in the context of the low ordering temperature, $T_{c}$ of this surface. At $600^{\circ} \mathrm{C}$ the top oxygen is in a disordered gas phase. Therefore, the corresponding LEED pattern can be modeled assuming a 50\% top oxygen occupancy of an unreconstructed surface, or a $p(1 \times 1)$ reconstruction, see Fig. 5a. The calculated $I$ - $V$ curves for $(00)$ and (01) beams (solid lines) are compared with the experiment (open symbols) in Fig. 5g. The best fit, $R_{p} \approx 0.4$, is obtained for the oxygen vacancy model with the first interlayer equal, within the error bar of the analysis, to the bulk value. Thus, on the basis of these results we can conclude that the model with randomly-distributed oxygen atoms, Fig. $5 \mathrm{a}$, is valid.

Fig. $3 b$ shows that the disordered oxygen vacancy states of the $\mathrm{Ce}\left(\mathrm{H}_{2}-\mathrm{H}_{2} \mathrm{O}\right)$ samples order into a $p(2 \times 2)$ phase at $\approx 300^{\circ} \mathrm{C}$. The satellite spots surrounding the principal (01) reflections are known to originate from incommensurate overlayers ${ }^{34}$. This strongly suggests the presence of an intermediate incommensurate phase, as predicted by the lattice gas theory for certain combinations of $\varphi_{n n}$ and $\varphi_{n n n}{ }^{33}$.

Finally, the sample $\mathrm{Ce}\left(\mathrm{H}_{2} \mathrm{O}\right)$ presents the most interesting case of a long-period structure. According to the lattice gas theory, $\times 4$ reconstruction requires strong long range coupling $\varphi_{t}$ in addition to the next nearest neighbor attraction ${ }^{29,33}$. We base our structure model on limited scanning tunneling microscopy (STM) data of heavily reduced $\mathrm{CeO}_{2}$ single crystal (001) termination with $\times 3$ reconstruction (Norenberg et al. ${ }^{35}$ ). The STM images show that the surface oxygen orders in row-like arrangements with $90^{\circ}$ rotational domains. A plausible structure that is consistent with the existence of $1 / 4$ fractional diffraction spots of the $\mathrm{Ce}\left(\mathrm{H}_{2} \mathrm{O}\right)$ sample is shown in Fig. 5 b. Here rows of the top layer oxygen order with $\times 4$ periodicity. The arrangement can be described as interleaving $\mathrm{Ce}$ and $\mathrm{O}$ terminated $p(4 \times 1)$ domains. The calculated diffraction pattern (Fig. 5b) which includes reflections from two kinds of rotational domains, is in qualitative agreement with the experiment (Fig. 2b). Currently, a quantitative analysis of the structure is not feasible due to computational limitations.

Our model explains the variety of surface structures of the (001) ceria termination by different couplings between the surface oxygen. The coupling strength is determined by the subsurface cerium atom structure which forms during transformation of (111)- terminated grains into much larger flat-top grains (see Fig. 1). This explains the stability of the reconstructions: the oxygen surface coverage is fixed by the charge neutrality and the coupling strength is determined by the practically immobile cerium lattice. The origin of the cerium atom ordering lies in the still unknown mechanism of surface reconstruction and simultaneous grain growth during the annealing process. In this work the oxidation state was measured after exposing the samples to air. Therefore, we cannot exclude a potential role of the surface $\mathrm{Ce}^{3+}$ ions in the initial reconstruction process. The in-situ XPS measurements of Ceria surfaces show a substantial surface reduction under conditions that correspond to the $\mathrm{Ce}^{4+}$ state of bulk ceria $^{36}$. It is likely that the difference between the three types of surface studied in this work can be traced to a different concentration of surface $\mathrm{Ce}^{3+}$ ions that existed during the annealing: one can reasonably expect the highest concentration of $\mathrm{Ce}^{3+}$ for the $\mathrm{Ce}\left(\mathrm{H}_{2}-\mathrm{H}_{2} \mathrm{O}\right)$ sample and practically zero concentration for the $\mathrm{Ce}\left(\mathrm{O}_{2}\right)$ sample.

Most intriguing is the role of water vapor in enabling the longrange coupling and formation of long-period surface order as seen in Fig. 2b. A tendency of water vapor towards the surface reduction has indeed been observed experimentally by XPS ${ }^{37}$. This effect cannot be easily explained by a strictly thermodynamic argument, since the Gibbs energy of bulk ceria reduction by water is positive. It has been argued that the absorbed water or hydroxyl molecules promote diffusion of bulk oxygen vacancies towards the surface, thus effectively generating $\mathrm{Ce}^{3+}$ ions ${ }^{37}$. This argument is indirectly supported by the recent work of Azimi et al. ${ }^{38}$, who reported an unusual hydrophobic character of a rare-earth oxide due to shielding of $4 \mathrm{f}$ orbitals of rareearth atoms by the outer $5 s^{2} p^{6}$ electrons. The observation implies that hydroxyl groups interact weakly with the surface cations and more strongly with the surface oxygen. This may explain the reducing action of water vapor and emergence of long-period ordering. A high-temperature DFT calculation, similar to the one performed for the non-polar (111) surfaces ${ }^{39}$, might provide a more quantitative description of the role of water vapor and $\mathrm{Ce}^{3+}$ ions in the hightemperature reconstruction.

We use a simple geometrical argument in order to visualize the effect of surface reconstruction on surface performance as a catalyst for YBCO nucleation. The experimental data shown in Fig. 4 suggest that the surface with the long-period reconstruction has the highest activity and yields epitaxial layers with best performance. The minimum size $c$-axis oriented YBCO nuclei would require a $0.38 \times$ $0.38 \mathrm{~nm}$ square attachment site, which is superimposed on the structural models in Fig. $5 \mathrm{a}-\mathrm{c}$. Both $c(2 \times 1)$ and $p(1 \times 1)$ domains have, on average, two oxygen vacancies per attachment site. In contrast, the long period $p(4 \times 1)$ domain offers a site free of desorbed oxygen, Fig. $5 \mathrm{~b}$. This model attributes the superior performance of the $\mathrm{Ce}\left(\mathrm{H}_{2} \mathrm{O}\right)$ sample to the lower energy of the YBCO nuclei, which results in smaller YBCO grain size. Reduced YBCO grain size has a positive effect on the intra-grain $J_{c}^{40}$ and the grain coupling ${ }^{41}$.

\section{Summary}

In summary, we show that post-annealing of a polar (001) Ceria surface can produce stable surface reconstructions, likely as a result of surface oxygen ordering. The reconstructions have markedly different surface activities, as demonstrated by the ability of the substrates to nucleate the high-temperature superconductor YBCO. The formation of desirable long-period reconstruction is related to a poorly understood high-temperature interaction of the (001) Ceria surface with water vapor. We suggest that in-situ surface X-ray diffraction could contribute to a better understanding of this effect and open new avenues for designing a highly active (001) surface.

\section{Methods}

Ceria film growth. The (001) $\mathrm{CeO}_{2}$ films were deposited on $10 \times 10 \mathrm{~mm}(001) \mathrm{YSZ}$ substrates (MTI Corporation) by pulsed laser deposition (PLD). The substrates were attached to a heater block by silver paste. Thereafter, the temperature of the substrate was raised to $600^{\circ} \mathrm{C}$ following the evacuation of the deposition chamber to $10^{-6}$ Torr. The deposition was carried out at 120 mTorr oxygen partial pressure, using a $5 \mathrm{~Hz}$ repetition rate of $\sim 3 \mathrm{~J}$ pulses, which corresponded to an average deposition rate of $0.2 \mathrm{~nm} / \mathrm{s}$. The film average film thickness was $70 \mathrm{~nm}$ as confirmed by scanning electron microscopy and X-ray reflectivity, see corresponding profiles in Fig. 1aS in the Supplementary Section.

Surface analysis. The bulk structure of the samples was characterized using the single crystal X-ray diffraction beamline X-18A at NSLS. Both in-plane and out-of-plane reflections were used to determine the corresponding lattice constants. The surface morphology of the samples was inspected by Atomic Force Microscopy (AFM) prior to and after the heat treatments. X-ray photoelectron spectroscopy (XPS) was performed in an ultrahigh vacuum (UHV) chamber equipped with a SPECS Phoibos $100 \mathrm{MCD}$ hemispherical analyzer. Al-K $\alpha \mathrm{X}$-ray radiation was used for the XPS studies. The background pressure of $5.0 \times 10^{-10}$ Torr was maintained throughout the XPS spectra acquisition. The X-ray reflectivity profiles were measured in a Rigaku Ultima III diffractometer in the parallel beam configuration. The Low Energy Electron Diffraction (LEED) experiment was performed using an Elmitec Low Energy Diffraction Microscope (LEEM) attached to the NSLS U5A beamline. We have determined that anneal at $600^{\circ} \mathrm{C}$ in UHV for 30 min removes the surface contaminants and permits acquisition of a usable LEED pattern. The $600^{\circ} \mathrm{C}$ sample temperature was maintained during the LEED experiment in order to avoid surface charging. The LEED patterns were acquired using a 5 micron aperture at the beam energies ranging from 10 to $45 \mathrm{eV}$ with $0.1 \mathrm{eV}$ intervals. A pattern from an unreconstructed $\mathrm{Ag}$ (001) surface was used as a calibration reference for the lattice parameter calculations. The Reflective High-Energy Electron Diffraction (RHEED) 
was performed using K-Space Associates differentially pumped RHEED system operating at $18 \mathrm{KV}$. The Raman microscopy was performed using an Alpha $300 \mathrm{R}$ Raman microscope (WITec).

Structure modeling. The diffraction patterns from the model structures were generated using the kinematic approximation. The structure models were further refined using the dynamic approach. Intensity versus electron energy $(I-V)$ curves were calculated using the Tensor LEED (TLEED) model $^{42}$. The phase shifts for $\mathrm{O}$ and Ce were evaluated from the muffin-tin potential calculated for $\mathrm{CeO}_{2}$ by means of the Barbieri-van Hove program package (http://www.icts.hkbu.edu.hk/surfstructinfo/ SurfStrucInfo_files/leed/). The LEED $I$ - $V$ curves of the $(00)$ and ( 01$)$ beams were used in the comparison with the experimental data in the 20-50 eV energy range. The imaginary part of the inner potential was set to $3 \mathrm{eV}$, whereas the real part was initially set to $10 \mathrm{eV}$ and then optimized in the search of the best fit with the experimental curves. A Debye temperature of $630 \mathrm{~K}$ was used to describe the vibrational effects. The Pendry reliability factor $\left(R_{p}\right)^{43}$ was used in the analysis to judge the agreement between experimental and calculated $I$ - $V$ curves. The computational approach at these low energies was validated on $I$ - $V$ curves of an $\mathrm{Ag}(001)$ recorded on the same instrument. The $I-V$ curves calculated for the $\mathrm{Ag}(001)$ surface yielded $R_{p}<0.2$.

Epitaxial growth on reconstructed surfaces. The effect of the surface reconstruction on the activity of the substrate was determined by growing epitaxial YBCO film via a fluoro-acetate method ${ }^{10}$. Fluorinated precursor layers were deposited on the substrate by spin-coating, which yielded $0.8 \mu \mathrm{m}$ thick YBCO film. The films were processed at $800^{\circ} \mathrm{C}$ in an atmosphere comprised of 300 mTorr oxygen and 300 mTorr water vapor in order to convert the precursor into an epitaxial YBCO layer ${ }^{44}$. The YBCO nucleation density was inferred from the optical and the Raman microscopy (Vitec Raman microscope) maps of mechanically polished film samples ${ }^{45}$. The critical current densities of the samples were measured by the magnetization method in external magnetic fields up to 3 Tesla using a Quantum Design magnetic properties measurement system.

1. Bibes, M., Villegas, J. E. \& Barthelemy, A. Ultrathin oxide films and interfaces for electronics and spintronics. Adv. Phys. 60, 5-84 (2011).

2. Reyren, N. et al. Superconducting interfaces between insulating oxides. Science 317, 1196-1199 (2007)

3. Ohtomo, A. \& Hwang, H. Y. A high-mobility electron gas at the $\mathrm{LaAlO}_{3} / \mathrm{SrTiO}_{3}$ heterointerface. Nature 427, 423-426 (2004).

4. Solovyov, V. F. et al. Nucleation of $\mathrm{ReBa}_{2} \mathrm{Cu}_{3} \mathrm{O}_{\mathrm{x}}(\mathrm{Re}=$ rare-earth $)$ during highrate metal-organic chemical vapor deposition growth. J. Appl. Phys. 110, 123904-123906 (2011)

5. Solovyov, V. F., Develos-Bagarinao, K., Li, Q., Qing, J. \& Zhou, J. Nature of $\mathrm{YBa}_{2} \mathrm{Cu}_{3} \mathrm{O}_{7}$ nucleation centers on ceria buffers. Supercond. Sci. Technol. 23, 014008 (2010).

6. Lee, S. et al. Template engineering of Co-doped $\mathrm{BaFe}_{2} \mathrm{As}_{2}$ single-crystal thin films. Nat. Mater. 9, 397-402 (2010).

7. Trovarelli, A. Catalysis by ceria and related materials. (Imperial College Press, 2002).

8. Goniakowski, J., Finocchi, F. \& Noguera, C. Polarity of oxide surfaces and nanostructures. Rep. Prog. Phys. 71, 016501 (2008).

9. Paier, J., Penschke, C. \& Sauer, J. Oxygen Defects and Surface Chemistry of Ceria: Quantum Chemical Studies Compared to Experiment. Chem. Rev. 113, 3949-3985 (2013).

10. Obradors, X. et al. Growth, nanostructure and vortex pinning in superconducting $\mathrm{YBa}_{2} \mathrm{Cu}_{3} \mathrm{O}_{7}$ thin films based on trifluoroacetate solutions. Supercond. Sci. Technol. 25, 123001 (2012).

11. Solovyov, V. F., Wiesmann, H. J., Wu, L. J., Zhu, Y. M. \& Suenaga, M. Kinetics of $\mathrm{YBa}_{2} \mathrm{Cu}_{3} \mathrm{O}_{7}$ film growth by postdeposition processing. Appl. Phys. Lett. 76, 1911-1913 (2000).

12. Thornton, J. A. High-Rate Thick-Film Growth. Ann. Rev. Mater. Sci. 7, 239-260 (1977).

13. Jiang, Y., Adams, J. B. \& van Schilfgaarde, M. Density-functional calculation of $\mathrm{CeO}_{2}$ surfaces and prediction of effects of oxygen partial pressure and temperature on stabilities. J. Chem. Pys. 123, 064701-064709 (2005).

14. Jacobsen, S. N., Madsen, L. D. \& Helmersson, U. Epitaxial cerium oxide buffer layers and $\mathrm{YBa}_{2} \mathrm{Cu}_{3} \mathrm{O}_{7-\delta}$ thin films for microwave device applications. J. Mater. Res. 14, 2385-2393 (1999).

15. Jacobsen, S. N., Helmersson, U., Erlandsson, R., Skarman, B. \& Wallenberg, L. R. Sharp microfaceting of (001)-oriented cerium dioxide thin films and the effect of annealing on surface morphology. Surf. Sci. 429, 22-33 (1999).

16. Van Hove, M. A., Weinberg, W. H. \& Chan, C. M. Low-energy electron diffraction: experiment, theory, and surface structure determination. (SpringerVerlag, 1986).

17. Thompson, C. V. Grain growth in thin films. Ann. Rev. Mater. Sci. 20, 245 (1990).

18. Solovyov, V. F., Develos-Bagarinao, K. \& Nykypanchuk, D. Nanoscale abnormal grain growth in (001) epitaxial ceria. Phys. Rev. B 80, 104102-104112 (2009).

19. Mullins, W. W. Theory of Thermal Grooving. J. Appl. Phys 28, 333-339 (1957).

20. Feng, X. et al. Converting Ceria Polyhedral Nanoparticles into Single-Crystal Nanospheres. Science 312, 1504-1508 (2006).
21. Coll, $\mathrm{M}$. et al. All chemical $\mathrm{YBa}_{2} \mathrm{Cu}_{3} \mathrm{O}_{7}$ superconducting multilayers: Critical role of $\mathrm{CeO}_{2}$ cap layer flatness. J. Mater. Res. 24, 1446-1455 (2009).

22. Coll, M., Pomar, A., Puig, T. \& Obradors, X. Atomically Flat Surface: The Key Issue for Solution-Derived Epitaxial Multilayers. Appl. Phys. Expr. 1, 121701 (2008).

23. Tasker, P. W. The stability of ionic crystal surfaces. J. Phys C: Solid State Phys. 12, 4977 (1979).

24. Lawrence, P. J. \& Parker, S. C. in Computer Modelling of Fluids Polymers and Solids Vol. 293 NATO ASI Series (eds Catlow, C. R. A., Parker, S. C. \& Allen, M. P.) Ch. 9, 219-248 (Springer Netherlands, 1989).

25. Conesa, J. Computer modeling of surfaces and defects on cerium dioxide. Surf. Sci. 339, 337-352 (1995).

26. Herman, G. S. Surface structure determination of $\mathrm{CeO}_{2}(001)$ by angle-resolved mass spectroscopy of recoiled ions. Phys. Rev. B 59, 14899-14902 (1999).

27. Bevan, D. J. M. \& Kordis, J. Mixed oxides of the type $\mathrm{MO}_{2}$ (fluorite) - $\mathrm{M}_{2} \mathrm{O}_{3}$ oxygen dissociation pressures and phase relationships in the system $\mathrm{CeO}_{2}-\mathrm{Ce}_{2} \mathrm{O}_{3}$ at high temperatures. J. Inorg. Nucl. Chem. 26, 1509-1523 (1964).

28. Murgida, G. E. \& Ganduglia-Pirovano, M. V. Evidence for Subsurface Ordering of Oxygen Vacancies on the Reduced $\mathrm{CeO}_{2}$ (111) Surface Using Density-Functional and Statistical Calculations. Phys. Rev. Lett. 110, 246101 (2013).

29. Landau, D. P. \& Binder, K. Phase diagrams and critical behavior of Ising square lattices with nearest-, next-nearest-, and third-nearest-neighbor couplings. Phys. Rev. B 31, 5946-5953 (1985).

30. Selke, W., Binder, K. \& Kinzel, W. Lattice gas models with competing interactions. Surf. Sci. 125, 74-93 (1983).

31. Demuth, J. E. \& Rhodin, T. N. Chemisorption on (001), (110) and (111) nickel surfaces: A correlated study using LEED spectra, Auger spectra and work function change measurements. Surf. Sci. 45, 249-307 (1974).

32. Fronzi, M., Soon, A., Delley, B., Traversa, E. \& Stampfl, C. Stability and morphology of cerium oxide surfaces in an oxidizing environment: A firstprinciples investigation. J. Chem. Phys. 131, 104701 (2009).

33. Binder, K. \& Landau, D. P. Square lattice gases with two- and three-body interactions: A model for the adsorption of hydrogen on Pd(100). Surf. Sci. 108, 503-525 (1981)

34. Kim, Y. J. et al. The growth of iron oxide films on Pt(111): a combined XPD, STM, and LEED study. Surf. Sci. 416, 68-111 (1998).

35. Norenberg, H. \& Harding, J. H. The surface structure of $\mathrm{CeO}_{2}(001)$ single crystals studied by elevated temperature STM. Surf. Sci. 477, 17-24 (2001).

36. DeCaluwe, S. C. et al. In Situ Characterization of Ceria Oxidation States in HighTemperature Electrochemical Cells with Ambient Pressure XPS. The Journal of Physical Chemistry C 114, 19853-19861 (2010).

37. Henderson, M. A., Perkins, C. L., Engelhard, M. H., Thevuthasan, S. \& Peden, C $\mathrm{H}$. F. Redox properties of water on the oxidized and reduced surfaces of $\mathrm{CeO}_{2}$ (111). Surf Sci 526, 1-18 (2003).

38. Azimi, G., Dhiman, R., Kwon, H.-M., Paxson, A. T. \& Varanasi, K. K. Hydrophobicity of rare-earth oxide ceramics. Nat. Mater. 12, 315-320 (2013).

39. Fronzi, M., Piccinin, S., Delley, B., Traversa, E. \& Stampfl, C. Water adsorption on the stoichiometric and reduced $\mathrm{CeO} 2(111)$ surface: a first-principles investigation. Phys. Chem. Chem. Phys. 11, 9188-9199 (2009).

40. Palau, A., Puig, T., Obradors, X., Feenstra, R. \& Gapud, A. A. Correlation between grain and grain-boundary critical current densities in ex situ coated conductors with variable $\mathrm{YBa}_{2} \mathrm{Cu}_{3} \mathrm{O}_{7-\delta}$ layer thickness. Appl. Phys. Lett. 88, 122502 (2006).

41. Solovyov, V. F., Li, Q., Wiesmann, H., Oleynikov, P. \& Zhu, Y. Strong influence of the $\mathrm{YBa}_{2} \mathrm{Cu}_{3} \mathrm{O}_{7}$ grain size on critical current densities of thick $\mathrm{YBa}_{2} \mathrm{Cu}_{3} \mathrm{O}_{7}$ layers made by a metal-organic deposition process. Supercond. Sci. Technol. 21, 125013-125018 (2008).

42. Van Hove, M. A. et al. Automated determination of complex surface structures by LEED. Surf. Sci. Rep. 19, 191-229 (1993).

43. Pendry, J. B. Reliability factors for LEED calculations. J. Phys C: Solid State Phys. 13, 937 (1980).

44. Solovyov, V., Dimitrov, I. K. \& Li, Q. Growth of thick $\mathrm{YBa}_{2} \mathrm{Cu}_{3} \mathrm{O}_{7}$ layers via a barium fluoride process. Supercond. Sci. Technol. 26, 013001 (2013).

45. Solovyov, V. F. \& Wiesmann, H. J. Application of low-angle polishing for rapid assessment of the texture and morphology of thick film $\mathrm{YBa}_{2} \mathrm{Cu}_{3} \mathrm{O}_{7}$ superconducting tapes. Physica C 467, 186-191 (2007).

\section{Acknowledgments}

This work was primarily supported by the US Department of Energy, Office of Basic Energy Science, Materials Sciences and Engineering Division, under contract no.

DEAC0298CH10886. X-ray reflectivity, AFM and XPS characterizations were carried the Center for Functional Nanomaterials, Brookhaven National Laboratory; LEED and XRD characterization was carried out at National Synchrotron Light Source (Beamlines X-18A, U5A), Brookhaven National Laboratory. The authors wish to thank AMSC Corp. for preparing the fluorinated precursor coatings; this work was supported by ARPA-E REACT program, award \# DE-AR0000190. We also would like to thank Ivo K. Dimitrov and Arnold Moodenbaugh for helpful comments and suggestions.

\section{Author contributions}

V.S. and Q.L. designed the study and wrote the manuscript. T.O. made the ceria thin films. V.S., J.T.S. and A.M. did LEED experiments, V.S. and X.T. did XPS, V.S. did XRD and X-ray 
reflectivity. D.N. did AFM, A.A. did numerical dynamic simulations. T.O. and Q.L. measured superconducting properties. L.J.W. did kinematic diffraction modeling. All the authors contributed to discussion on the results for the manuscript.

\section{Additional information}

Supplementary information accompanies this paper at http://www.nature.com/ scientificreports

Competing financial interests: The authors declare no competing financial interests.
How to cite this article: Solovyov, V.F. et al. Highly efficient solid state catalysis by reconstructed (001) Ceria surface. Sci. Rep. 4, 4627; DOI:10.1038/srep04627 (2014).

This work is licensed under a Creative Commons Attribution-NonCommercialNoDerivs 3.0 Unported License. The images in this article are included in the article's Creative Commons license, unless indicated otherwise in the image credit; if the image is not included under the Creative Commons license, users will need to obtain permission from the license holder in order to reproduce the image. To view a copy of this license, visit http://creativecommons.org/licenses/by-nc-nd/3.0/ 